\title{
ON INDETERMINISM, CHAOS, AND SMALL NUMBER PARTICLE SYSTEMS IN THE BRAIN
}

\author{
EDWIN R. LEWIS \\ Department of Electrical Engineering and Computer Science \\ University of California, Berkeley, CA 94720-1770, USA \\ lewis@eecs.berkeley.edu \\ RONALD J. MACGREGOR* \\ Department of Aerospace Engineering Sciences, University of Colorado \\ 38 Rock Ridge Dr. NE, Albuquerque, NM 87122-2007, USA \\ ronaldjmacgregor@msn.com
}

Received 6 March 2006

Accepted 14 April 2006

\begin{abstract}
This paper presents rational, theoretical, and empirical grounds for doubting the principle of determinism in nature and in the brain, and discusses implications of this for free will and the chaos model of the brain. Small number particle systems are practically indeterministic and may be intrinsically indeterministic. Determinism in nature has often been taken to preclude free will. Strict determinism is a concept frequently applied to systems theory, establishing, e.g., the uniqueness of state-space trajectories. In order to consider determinism as a law of nature, however, one must be able to subject it to empirical tests. Presently, one is not able to and whether this can be shown to enable free will or not is not clear. It does remove, at least for the present, determinism itself as a rationale for precluding free will. The work partially supports the chaos model, but undermines the computational computer metaphor of brain function.
\end{abstract}

Keywords: Determinism; free will; consciousness; brain; chaos model.

\section{Introduction}

The mind-body question has been deadlocked in Western Philosophy for several centuries between monists, who consider both the experiential qualities of the mind and the physical (material) qualities of the brain as substantively one (i.e., the former arising from the latter), and dualists who see the two as distinct realms which stand in significant but obscure relation to each other. Within the (nearly) universally presumed determinism of the pre-twentieth century and macroscopic twentieth-century physical science, brain-mind monism has been taken to imply a 
strict physically-based determinism of all experiential qualities by the brain, thereby denying any degree of free will and reducing consciousness to a functionless, useless epiphenomenon. This view can be summarized by stating that, regarding free will, the universe of possibilities comprises the union of FREE WILL and DETERMINISM and that the intersection of FREE WILL and DETERMINISM is null. Regarding material causality in the realm of natural science, the universe of possibilities comprises the union of DETERMINISTIC CAUSALITY and STOCHASTIC CAUSALITY, where the intersection of the two is again null. Recent efforts in brain theory and the philosophy of mind are arguing for middle ground between the classical monist-dualist polarity. This paper argues, on rational and empirical grounds, that determinism cannot be presently taken as a law of material nature even for macroscopic systems, and therefore should not be taken, by monists, as a constraint on hypotheses concerning free will. For systems involving colliding particles, such as molecules in some form of fluid, a rational alternative to determinism is the molecular chaos posited by Boltzmann, which corresponds to assumptions made by both Maxwell and Gibbs as well. With respect to properties involving large numbers of colliding particles, such systems may appear to be deterministic. For small-number particle systems, even this appearance is gone. This paper argues that small-numbers processes are ubiquitous in the brain and reviews experimental findings in situations known to involve especially small numbers, leading to behavioral or perceptual responses that are conspicuously unpredictable. It is argued that the ubiquitous small-numbers processes in the brain lead to an inherent, conspicuous indeterminism in brain processes. The question that arises, then, is whether or not this provides an opening for free will in brain function. Does stochastic causality somehow enable free will? At the very least, it enables the philosopher to look beyond the rejection of free will simply on the basis of determinism. Pursuant to these issues, the paper probes briefly the fundamental principles and questions that underlie both the implications of this and other related work. This discussion necessarily involves the nature and interrelations of natural science, free will, and consciousness. A central purpose of this paper is to encourage more active cultivation of this area by neuroscientists. The bulk of the paper focuses on the rational and empirical basis of effective indeterminism in macroscopic physics, both generally and in the brain and its implications for models of the brain. The work partially supports the chaos model but seriously weakens and restricts the computational model.

\section{Philosophical and Physical Analysis}

\subsection{Causality and the scope of natural science}

Material causality, as we think of it today, has its roots perhaps in the early Enlightenment, with materialistic philosophers such as Thomas Hobbes. When Hobbes posited that for every event there is a cause, he associated cause with matter. If one defines matter to include all things comprising mass and/or energy and existing 
in space-time, his view would be consistent with modern physics. It also provides the definition of the scope of modern natural science - natural science is limited to studies of the nature of matter, the nature of space-time, and the bases of material causality. Even today, Hobbes' restatement of Descartes' epistemological foundation, "I think therefore matter is capable of thought", joins evolution (in its broadest sense, including astrophysical evolution) to form the portcullis at the gate separating the realm of natural science from that of faith-based philosophies. The issue at hand in the first part of this paper, however, is not Hobbes' response to Descartes' dualism. It is the ultimate nature of the mapping from cause to effect is it deterministic or is it probabilistic? Can one know? In this section, quantum mechanics, wave functions, and Heisenberg's uncertainty principle will all be set aside; and the focus will instead be placed on the demands of determinism even on macroscopic phenomena.

\subsection{Strong inference, the epistemology of natural science}

Since the time of Galileo, Descartes and Bacon, who participated in the overthrow of the Western European version of Aristotelian philosophy, natural scientists have looked to nature itself, rather than books, for knowledge of nature. The empirical approach practised by the new generations of natural scientists led to the publication of a small set of fundamental laws of physics. These were compact descriptions of the elementary behavior of matter, based on the new paradigm of strong inference (see Refs. 29, 31 and 33). The elements of strong inference include (1) careful observation of some aspect of matter in space-time and collection of data, (2) reflection on the observations, leading to generalizations (creation of tentative hypotheses), (3) reflection on the hypotheses and their implications, leading to identification of experiments or observations that would test them most severely, (4) carrying out those experiments or observations, (5) elimination or modification of failed hypotheses and further testing of surviving hypotheses. The earliest members of the new generation, led arguably by Isaac Newton, incorporated mathematics into the process of strong inference. They constructed many of their tentative laws in the forms of mathematical functions, and then using mathematical analysis to deduce the implications of those laws. The new paradigm required that these empirically-based laws be placed always in jeopardy, subjected to continued verification with real data, real observations. Quintessentially, they must be empirically testable.

The paradigm of strong inference could thus be applied only to properties and phenomena that were currently accessible to empirical observation. As Locke stated explicitly, only such phenomena could be considered knowable by this new generation of scientists. Their universal acceptance of Newton's synthesis established precedence for two avenues of observation: (1) direct observation of an elementary behavior, and (2) indirect observation of hypothesized elementary behavior through its implications regarding observable phenomena. The hypothetical laws of motion (involving conservation and transfer of translational momentum) were 
easily subjected to repeated direct observation over a wide variety of situations, such as those described by Newton in The Principia (see Ref. 11). The hypothetical (inverse-square) law of gravity, on the other hand, was a nonlinear curve fitted only to two direct observations of the acceleration owing to earth's gravity, that at the surface of the earth itself and that at the moon. Combined with the laws of motion, however, the law of gravity implied Kepler's laws, and it did so precisely. In other words, it predicted the observed properties of planetary and lunar orbits as Kepler had generalized them from existing data. There is no doubt that this fact was crucial to its widespread acceptance. Knowing what we now do about the ambiguities in any inverse problem, however, we must consider the second avenue as the less certain of the two. Nonetheless, it is the avenue followed by modern physicists when they consider the fine-structure of space-time, which is central to the issue of determinism. Ultimately, it may prove to be the only avenue open to us; we may be destined to know the properties of space-time only through their implications.

\subsection{The deterministic ideal}

According to the deterministic ideal, which is a purely mathematical concept, if one knows the laws governing the dynamics of a system, and if one knows precisely the initial state of the system, along with the precise history of subsequent perturbations of that system, then one can precisely predict the state of the system at any time. It is this precision that is so demanding, even on macroscopic systems. The sense that macroscopic physical systems might be deterministic arises perhaps from the predictability of planetary and lunar orbits and rotation. In the major planetary elements of the solar system, the rate of energy dissipation by tidal phenomena and the like is exceedingly small in comparison to the total energy of planetary kinetics, and collisions of planetary objects are rare, making the entire system nearly conservative and highly predictable. In the following paragraphs, we shall consider a completely different kind of system, one in which instability leads to the rapid divergence of state trajectories from their initial neighborhood, no matter how precisely defined that neighborhood is.

\subsection{Kinetics of two non-deformable spheres undergoing collisions}

Consider the situation depicted in Fig. 1. In a gravity-free environment, there are two identical spheres, perfectly rigid, spherical and smooth, that undergo perfectly elastic collisions with one another and with perfectly rigid and smooth, vertical walls. Assuming that these perfections dictate that the forces resulting from the collision of the two spheres are aligned perfectly along the line passing through their centers, and that the forces resulting from the collision of a sphere and a wall are aligned perfectly perpendicular to the wall. Thus, if the two spheres were initially moving precisely along their line of centers, and if their line of centers were aligned precisely perpendicular to each wall, one would predict that their motion would forever remain on that same line. So far, nothing in this model is inherently 


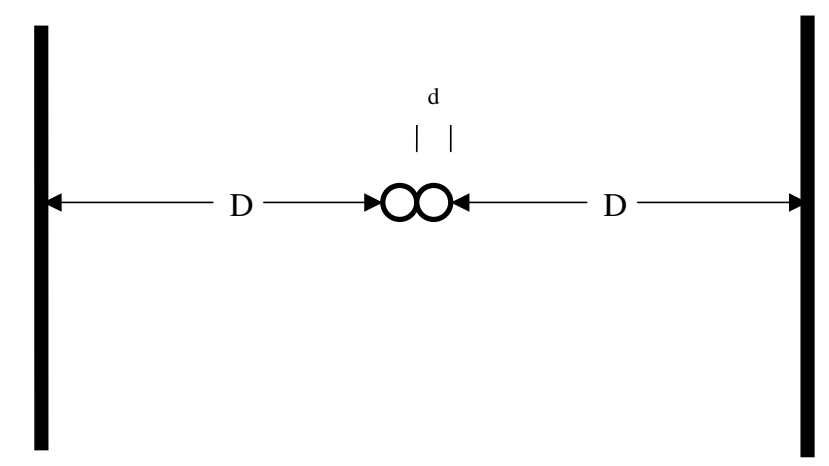

Fig. 1. A thought model for computing some demands on determinism: two spheres collide with each other and with opposing vertical walls.

inconsistent with the deterministic ideal. The hypothetical situation is, however, exceedingly unstable. The slightest error in alignment will lead to components of motion perpendicular to the line of centers, and each successive collision of the two spheres will accelerate those components. Assuming that the magnitudes of the initial velocities are precisely equal, and that the initial velocity vector of each sphere is aligned perfectly perpendicular to the walls, but that the two initial velocity vectors are displaced from one another by a miniscule error, $\varepsilon_{0}$, one can invoke Newton's second and third laws, the definition of work, the equating of kinetic energy to work, and the first law of thermodynamics to estimate the relationship between the magnitude of the error and the number of subsequent collisions.

Taken together, Newton's second and third laws tell us that the translational momentum of the system is conserved. Thus,

$$
\begin{aligned}
& \mathrm{m} v_{x 1}+\mathrm{m} v_{x 2}=\mathrm{m} v_{x 1}(0)+\mathrm{m} v_{x 2}(0)=0 \\
& \mathrm{~m} v_{y 1}+\mathrm{m} v_{y 2}=\mathrm{m} v_{y 1}(0)+\mathrm{m} v_{y 2}(0)=0
\end{aligned}
$$

where the $x y$ plane is that plane containing the two initial velocity vectors, the $x$-axis being perpendicular to the walls and the $y$-axis being parallel to the walls; $\mathrm{m}$ is the mass of each sphere; $v_{x 1}$ and $v_{x 2}$ are the velocity components of spheres 1 and 2 respectively, in the $x$ direction; $v_{y 1}$ and $v_{y 2}$ are their velocity components in the $y$ direction. According to the statement of the model, the initial velocity vectors, $v_{i j}(0)$, at the instant immediately prior to the first collision are related as follows:

$$
\begin{aligned}
& v_{x 1}=-v_{x 2}=v_{0} \\
& v_{y 1}=v_{y 2}=0 .
\end{aligned}
$$

Newton's second and third laws also tell us that, on colliding with one another, the two spheres will undergo equal but opposite accelerations. Thus, after each such collision,

$$
\begin{aligned}
& v_{x 1}=-v_{x 2} \\
& v_{y 1}=-v_{y 2} .
\end{aligned}
$$


228 Lewis \& MacGregor

The definition of the work tells us that

$$
d W_{x}=f_{x} d x
$$

where $d W_{x}$ is the incremental work done parallel to the $x$-axis on a particle; $f_{x}$ is the force applied to the sphere parallel to the $x$-axis, and $d x$ is the incremental distance through which the force acts on the particle. Combining this with Newton's second law and defining the $x$-component $\left(E_{x}\right)$ of translational kinetic energy as the total accumulation of work on the sphere, parallel to the $x$-axis, one finds

$$
E_{x}=\frac{1}{2} m v_{x}^{2}
$$

Similarly,

$$
E_{y}=\frac{1}{2} m v_{y}^{2}
$$

The first law of thermodynamics tells us that the kinetic energy of the system is conserved. Thus,

$$
\frac{1}{2} \mathrm{~m} v_{x 1}^{2}+\frac{1}{2} \mathrm{~m} v_{x 2}^{2}+\frac{1}{2} \mathrm{~m} v_{y 1}^{2}+\frac{1}{2} \mathrm{~m} v_{y 2}^{2}=m v_{0}^{2} .
$$

Combining (2.3) and (2.7), one finds that

$$
\begin{aligned}
& v_{x 1}^{2}+v_{y 1}^{2}=v_{0}^{2} \\
& v_{x 2}^{2}+v_{y 2}^{2}=v_{0}^{2} .
\end{aligned}
$$

As long as the magnitude of $v_{y 1}$ and $v_{y 2}$ remains very much less than the magnitude of $v_{x 1}$ and $v_{x 2}$, the latter will be very well approximated by

$$
\operatorname{mag}\left(\frac{v_{x i}}{v_{0}}\right)=1-\frac{1}{2} \operatorname{mag}\left(\frac{v_{y i}^{2}}{v_{0}^{2}}\right)
$$

and the magnitude of $v_{x 1}$ and $v_{x 2}$ will remain exceedingly close to that of $v_{0}$.

After each collision between the two spheres, a small amount of kinetic energy will have been transferred from translational motion in the $x$ direction to translational motion in the $y$ direction. The amount will depend on the misalignment, $\varepsilon$, of $v_{x 1}$ and $v_{x 2}$ at the instant of collision. Taking the magnitudes of $v_{x 1}$ and $v_{x 2}$ to be equal to that of $v_{0}$, and recalling that the impact force is aligned precisely along the line of centers of the two particles, one finds

$$
\begin{aligned}
\Delta v_{y 1}(i) & =-\Delta v_{y 1}(i)=v_{0} \sin \left(2 \theta_{i}\right) \\
\sin \theta_{i} & =\frac{2 \varepsilon(i)}{\mathrm{d}}
\end{aligned}
$$

where $\Delta v_{y}(i)$ is the change in the $y$-direction velocity as a result of the $i$ th collision, $\mathrm{d}$ is the particle diameter, and $\varepsilon(i)$ is the misalignment of $x$-direction velocity 
components at the instant of the $i$ th collision. Taking $\varepsilon(i)$ to be exceedingly small in comparison with d, one can simplify (2.10) as

$$
\Delta v_{y 1}(i)=-\Delta v_{y 2}(i)=\frac{4 \varepsilon(i)}{\mathrm{d}} v_{0} .
$$

After the $i$ th collision,

$$
v_{y 1}(i)=-v_{y 2}(i)=v_{y 1}(i-1)+\frac{4 \varepsilon(i)}{\mathrm{d}} v_{0} .
$$

As long as $\varepsilon(i)$ remains exceedingly small in comparison with $\mathrm{d}$, the time, $\mathrm{T}$, between collisions will be exceedingly close to $\mathrm{D} / v_{0}$. In that case, the growing error will be well approximated by

$$
\varepsilon(i)=\varepsilon(i-1)+2 \frac{v_{y 1}(i-1)}{v_{0}} \mathrm{D} .
$$

Written in normalized, canonical form, (2.12) and (2.13) become

$$
\begin{array}{r}
\widehat{v_{y 1}}(i)-\widehat{v_{y 1}}(i-1)-4 \hat{\varepsilon}(i)=0 \\
\hat{\varepsilon}(i)-\hat{\varepsilon}(i-1)-2 \widehat{D} \widehat{v_{y i}}(i-1)=0
\end{array}
$$

where

$$
\begin{aligned}
\widehat{v_{y 1}}(i) & =\frac{v_{y 1}(i)}{v_{0}} \\
\hat{\varepsilon}(i) & =\frac{\varepsilon(i)}{\mathrm{d}} \\
\widehat{D} & =\frac{\mathrm{D}}{\mathrm{d}} .
\end{aligned}
$$

One can derive (2.14) purely geometrically. For each collision and each sphere, take the angle of reflection relative to the line of centers (at the instant of collision) to be equal to the angle of incidence.

The solution to (2.14) is

$$
\begin{aligned}
\hat{\varepsilon}(i) & =\frac{r_{1}-1}{r_{1}-r_{2}} \hat{\varepsilon}_{0} r_{1}^{i-1}-\frac{r_{2}-1}{r_{1}-r_{2}} \hat{\varepsilon}_{0} r_{2}^{i-1} ; \quad i \geq 1 \\
r_{1} & =1+4 \widehat{D}+2 \sqrt{2 \widehat{D}+4 \widehat{D}^{2}} \\
r_{2} & =1+4 \widehat{D}-2 \sqrt{2 \widehat{D}+4 \widehat{D}^{2}} .
\end{aligned}
$$

The mean-free-path for air molecules at $1 \mathrm{~atm}$ pressure and $300 \mathrm{~K}$ is approximately 300 times the diameters of those molecules. For our gedanken model, therefore, selecting $\mathrm{D} / \mathrm{d}$ to be 100 seems thoroughly reasonable. In that case,

$$
\begin{aligned}
r_{1} & \cong 800 \\
r_{2} & \cong 0 \\
\hat{\varepsilon}(i) & \cong(800)^{i-1} \hat{\varepsilon}_{0} .
\end{aligned}
$$

This approximation will be valid as long $\varepsilon(i) / \mathrm{d}$ is much less than 1 . Consider $\varepsilon(i) / \mathrm{d}=0.01$ to be a threshold. For smaller errors, (2.17) is valid. Once the threshold 
is reached, the very next collision will cause the misalignment to be larger than the radius of each sphere, and the spheres will no longer collide on each pass between the walls. We can now ask how small the initial misalignment must be in order for the current misalignment to remain below threshold through the Nth collision. The answer is

$$
\hat{\varepsilon}_{0}<\frac{0.01}{800^{N-1}} .
$$

According to (2.18), if d were $1.0 \mathrm{~m}$, then to guarantee that the system remain below threshold through just the 12 th collision, the initial misalignment would have to be approximately 1 Planck length (approximately $1.6 \times 10^{-35} \mathrm{~m}$ ) or less. Considering the uncertainty among modern physicists concerning the nature of space at the level of the Planck length, one need go no further to place the deterministic ideal beyond the realm of that which is known, through strong inference, even in a classical, macroscopic situation such as this. Based on our concept of real numbers, which are usually defined to form a compact set, (2.18) is not constrained by the nature of space; it will carry us as far as we want to go. To guarantee that the system remain below threshold through the 35th collision, for example, the initial misalignment would have to be less than one-googleth of a meter $\left(10^{-100} \mathrm{~m}\right)$. A photon with a wave length of that magnitude would have energy equal to approximately $2 \times 10^{75} \mathrm{~J}$. That would be the energy available from approximately $10^{28}$ solar masses, or approximately $10^{16}$ Milky-Way galaxies, all concentrated in one photon.

\subsection{Mathematics is not natural science}

Mathematicians are divided over the source of the real numbers. Were they invented by humankind, or were they inherent in nature and merely discovered by humankind? If the latter were true, then perhaps the knowledge of the universe lay hidden in their properties and relationships. Perhaps modern Aristotelians can replace the ancient Euro-Aristotelian texts with the book of real analysis, and search for the secrets of nature. The modern Baconian and Lockean, on the other hand, would take his or her laws of nature from observations of nature itself. To such a person, mathematics is and must remain an axiomatic science, and not a natural science; and the fact that one can take any two real numbers, no matter how close together, and write the names of an infinite number of other numbers that lie between them, provides no implications whatsoever regarding the properties or fine-structure of space-time.

\subsection{The deterministic ideal is not a law of physics}

From the gedanken experiment with two spheres, which was based on the simplest (best case) geometries and on laws extracted by strong inference from nature itself, we know that it would require more energy than is currently posited for the entire universe in order to resolve the initial state of the system with precision sufficient 
to predict its behavior after just 35 collisions. This eliminates the possibility of applying strong inference to the deterministic ideal through the first path - direct observation. The current controversy between string theorists and quantum gravity advocates, regarding the fine structure of space-time (e.g., see Refs. 19, 26 and 44), tells us that we are currently unable to apply strong inference to the deterministic ideal, even through the second, less-certain path of indirect observation (deduction and testing of implications). The deterministic ideal, therefore, cannot be currently considered a law of physics in the traditional sense.

\subsection{Real implications, molecular chaos}

Scaling the geometry of the hard-sphere model to the dimensions of air molecules, one obtains comparable results. The diameter of the nitrogen molecule $\left(\mathrm{N}_{2}\right)$ is estimated to be approximately $3 \times 10^{-10} \mathrm{~m}$. At $300 \mathrm{~K}$ and $1.0 \mathrm{~atm}$, its mean free path is estimated to be approximately $9 \times 10^{-8} \mathrm{~m}$. At that same temperature, its root-mean-square velocity should be approximately $500 \mathrm{~m} / \mathrm{s}$, making its collision rate approximately $5 \times 10^{9}$ per sec. The error threshold established in this scaled model would be reached in approximately 9 collisions, if the initial displacement were 1 Planck length, with the number of collisions occurring in approximately 2 nanoseconds. Although the hard-sphere model is frequently invoked in the kinetic theory of ideal gases, for diatomic molecules in air $\left(\mathrm{N}_{2}\right.$ and $\mathrm{O}_{2}$ ) a dumbbell-shaped model (two hard spheres with a rigid connection between them) better fits the observed physics (e.g., the ratio of heat capacity at constant pressure to that at constant volume). Given the premises about impact forces stated for the hard-sphere model itself, it is apparent that the dumbbell particle can accumulate energy in its translational velocity, along each of the three orthogonal axes and in its rotational velocity about each of two orthogonal axes. The outcome of each collision between pairs of these idealized molecules will depend not only on each molecule's five components of velocity, but also on the rotational orientation of each molecule at the instant of collision. The kinetic energy that was initially restricted to the translational velocity orthogonal to the walls in the hard-sphere model, will rapidly become distributed among all five velocity components, in what Boltzmann termed as molecular chaos.

\subsection{Statistical mechanics and the laws of large numbers}

Faced with this reality, to create computationally-manageable, predictive models of systems comprising colliding particles such as gas molecules, the early kinetic theorists, such as Gibbs, Boltzmann and Maxwell (e.g., see Ref. 47), invoked a statistical approach, i.e., inventing the new field of statistical mechanics. This development in itself does not imply lack of determinism. However, it does provide a foundation in mechanics for collective properties of such a system, properties such as its volume $(\mathrm{V})$, its density $(\rho)$, its temperature $(\mathrm{T})$, its energy $(\mathrm{E})$ and its pressure $(\mathrm{P})$, and for 


\section{Lewis 83 MacGregor}

the relationships among them. It also indicates that when any of those properties is treated as a dependent variable, instead of a fixed variable, it is the spatiotemporal mean of a very large set of random numbers. Determinism in any relationship among those properties is therefore an illusion. Consider, for example, a system comprising two rigid-walled vessels, each with internal volume $\mathrm{V}_{o}$, sharing a common wall with a small hole. Initially, each vessel contains $n$ particles of an ideal, monatomic gas. Assuming that the particles collide occasionally, but otherwise move independently, and that each particle is equally likely to be in vessel A or vessel B, at any instant, therefore, the division of particles between the two vessels can be considered the outcome of $N=2 n$ Bernoulli trials, each with $p=1 / 2$ (the probability that the particle is in A) and $q=1 / 2$ (the probability that the particle is in B). Let $\chi(t)$ be the number of particles in $\mathrm{A}$ at time $t$; let $m(\chi)$ be its expected value; and let $\sigma(\chi)$ be its standard deviation [the root-mean-square of the difference between $\chi$ and $m(\chi)]$.

$$
\begin{aligned}
& m(\chi)=p N=n \\
& \sigma(\chi)=\sqrt{p q N}=\sqrt{n / 2} .
\end{aligned}
$$

Instantaneous observations of $\chi$ can thus be considered to be noisy, $\sigma(\chi)$ being the amplitude of noise component. The density, pressure, and the temperature of the gas contained in vessel A will vary accordingly, exhibiting comparable noisiness.

$$
\begin{aligned}
\rho & =\left(\mu / \mathrm{N}_{\mathrm{A}} \mathrm{V}_{0}\right) \chi \\
P & =\left(2 E / 3 \mathrm{~V}_{0}\right) \chi \\
T & =\left(2 E / 3 \mathrm{k}_{\mathrm{b}}\right) \chi^{-1}
\end{aligned}
$$

where $\mathrm{k}_{\mathrm{b}}$ is the Boltzmann constant (the molar gas constant divided by Avogadro's number, $\mathrm{N}_{\mathrm{A}}$ ) and $\mu$ is the molar weight of the gas. When interpreting (2.20), one must remember that according to the thought model we are using here, $E$, the total kinetic energy of the $\chi$ particles currently in vessel $\mathrm{A}$, will also be a random variable.

One sees from Table 1 that the relative importance of the noise diminishes as the particle population increases. When the numbers of particles are very large, the noisiness in the relationships in (2.20) will very likely be below that of available laboratory instruments, giving the relationships themselves the appearance of being deterministic.

Table 1. Relative noise vs mean number of particles.

\begin{tabular}{ccccc}
\hline$m(\chi)$ & 10 & $10^{3}$ & $10^{5}$ & $10^{23}$ \\
\hline$\sigma(\chi) / m(\chi)$ & 0.22 & $2.2 \times 10^{-2}$ & $2.2 \times 10^{-3}$ & $2.2 \times 10^{-12}$ \\
\hline
\end{tabular}




\subsection{The business of neurons often is conducted in small-number situations}

When one thinks of neuronal processes, one is immediately confronted with populations of discrete elements, i.e., discrete g-protein molecules, discrete ion channels, discrete ions, discrete synapses, and discrete packets of synaptic transmitter. Physiological evidence suggests that these elements are frequently engaged in small numbers during normal neuronal operation. Among the most conspicuous neural examples of small-number situations are those that occur in chemoreceptors and photoreceptors, where the capture of a single particle (single photon or single molecule) can produce a physiological response in a single receptor cell (see Refs. 1, 3, 17, 27 and 38). The number of particles required for perceptual response, however, may be considerably greater than one. In the celebrated sex pheromone detection system of the silk-worm moth (Bombyx mori), for example, Kaissling and his colleagues showed that one-second stimulus bursts delivering an average of 700 molecules of the pheromone bombykal to the animal's antenna elicited behavioral response in the male moth, $20 \%$ of the time [27]. An average of approximately $25 \%$ of these molecules produced nerve impulses (spikes) at one spike per molecule. The actual number of spikes $(\chi)$ elicited by each stimulus, was of course a random number, and it was observed to follow a Poisson distribution. In that case, $\sigma(\chi) / m(\chi)$ would be approximately 0.075 . One might argue that this $7.5 \%$ noise level simply reflects an underlying deterministic process that we are ignoring. Ignoring particle wave functions and Heisenberg uncertainty, on the other hand, the modern Baconian and Lockean physicist would be compelled to attribute the noise to Boltzmann's molecular chaos and to argue that we are presently incapable of knowing whether or not the underlying process is deterministic.

The noise level is even more significant in the perceptual detection of the nearthreshold light flashes during scotopic vision. For a $60 \%$ hit rate (human subjects reported sensing light flashes $60 \%$ of the time they were presented), Hecht et al. [17] estimated that an average of about nine photons were captured by the retina. With $\chi$ being the number of captured photons, the relative noise level, $\sigma(\chi) / m(\chi)$, would have been approximately 33\%. Most modern psychophysicists would repeat the experiment with random forced choice trials, during which each of the light flash was or was not presented. For the trials without light flashes, the investigator would measure the false-alarm rate instead (the percentage of trials during which the subject reported sensing a flash when none was present). False alarms occur evidently, owing to the presence of noise inherent in the sensory system itself, operating far from $0 \mathrm{~K}$. In the case of the retina, isomerization of photopigment molecules is induced either by photon absorption or by accumulation of sufficient thermal energy (a sufficiently large share of the local kinetic energy in the receptor cell). This thermal isomerization mimics the effect of light, creating retinal output even in the absence of light [2]. Psychophysicists have found that, in response to subtle suggestion, the human subject can adjust his/her strategy in the face of this internal noise, 
leading to a change in hit rate and a concomitant change in false-alarm rate. As one would expect, when the adjusted hit rate is higher, so is the adjusted false-alarm rate, and vice versa. This suggests that the adjustment involved the shifting of an internal decision (perceptual) threshold or criterion. This, in turn, brings us to that powerful tool of modern sensory biology, that of signal detection theory [14]. Modern sensory biologists frequently speak of the sensitivity of a system not in terms of an absolute threshold, but in terms of that theory's receiver operating characteristic and discriminability index, $d^{\prime}$. It is interesting to contemplate the costs that might be associated with misses (the counterparts of hits) and those associated with false alarms in various situations, and to ponder the impact of natural selection on internal thresholds or criteria. Would the moth's internal threshold for the sex pheromone be altered by the presence of subthreshold (subliminal) ultrasonic emissions from a bat, and would this be genetically programmed? The ability of the human subject to adjust his/her internal threshold suggests that molecular chaos and randomness may not preclude free will. Perhaps, in fact, they make free will possible.

\section{Discussion}

The body of this paper has made and tried to justify the fundamental claim that the principle of determinism in classical macroscopic physics is an article of faith and not a demonstrable law of material nature. This applies to the inherent, fundamental nature of matter and space-time, not merely to practical difficulties of measurement, unavoidable disturbances, and the like. The ultimate judgment in any case appeals to empirical verification. The question of determinism may ultimately prove amenable to clever experimentation and resolution. In the meantime, however, determinism should not be imposed as a constraint on hypotheses about nature. Removal of this constraint may significantly influence how we think about the physical universe, our place in it, and, for example, such important obscure regions as the brain. Deterministic models, on the other hand, may be thoroughly predictive for nearly conservative systems of weakly-connected (e.g., by gravity), non-colliding particles and for the colligative properties of systems involving very large numbers of colliding or strongly-connected particles (as in gasses, liquids, and solids). This fidelity of deterministic models will fail whenever the numbers of colliding or strongly-connected particles becomes small.

Our rational and theoretical arguments have suggested that small numbers systems are certainly indeterministic in an observational sense, and may be indeterministic in an intrinsic sense. Small number circumstances should apply to vast numbers of synapses and synaptic junctions throughout the brain and to vast numbers of other particles such as receptor molecules. The body of physiological and biophysical literature identifies a number of specific recognizable instances of random behavior in such receptor molecules and is suggestive of its widespread occurrence. This is important because a strict determinism seems to render free will an illusion and consciousness itself an ineffectual epiphenomenon. Many hold the view that individual 
free will is an undeniable quality of human existence and that this requires some non-traditional modification of traditional physics, in order to reconcile observed brain-mind relations beyond a not completely satisfying totally separative dualism.

This section will explore this possible avenue to free will through the consideration of a stochastic molecular chaos model for brain activity. We will find that the model suggests a plausible description of brain activity, which leaves an "open door" for selective choices of free will. To develop this model further, of course, requires more explicit presumptions or characterizations regarding the nature and interrelations of the triad of physical science, free will and consciousness. This is necessarily more speculative and unavoidably brings additional non-traditional factors into play. This is beyond the scope of the molecular chaotic model in itself, but the chaos model can be usefully combined with more detailed hypotheses regarding physical relations of free will and consciousness. Also, the possible identification of indeterminism, particularly to small number subsystems, is a convenient suggestive clue to localization. This discussion will be presented within a consideration of possible intermediate views of consciousnessbrain relations between the classical poles of deterministic monism and separative dualism.

The statistical molecular chaos identified here for small number brain subsystems, whether only practical or both practical and intrinsic, allows the application of useful and descriptive statistical modeling techniques [13]. Indeterminism seems an inherent characteristic of at least some significant portion of brain activity.

\subsection{Free will, consciousness, and the brain}

Free will is necessarily intimately interrelated with consciousness and the brain, and herein reside all the obscurities and mysteries of the subject. Consciousness holds a rich matrix of qualities, internal or externally directed, in passive reception or active assertion. It is perhaps most clearly manifested in times of quiet detached reflection or total intensely focused engagement with immediate highly threatening situations. Nonetheless, consciousness and free will are both intimately associated with unconscious operations indicated in both consciousness and brain study. The term "consciousness system" can describe either the set of all manifestations of consciousness and its intimate unconscious associates, or a unified integrated system supposed to underlie these [42]. We can instructively outline a model of such a unified consciousness system as a triumvirate of inner reflective space, sensory experience, and partially free will. Such a model includes qualities that are "experiential" and of "experience" (the hallmark of "conscious") and those that are "mental" and of "mind" (the hallmark of "cognitive"), but is significantly larger than either or both, and the two subsets only partially overlap. The recognition of both the important non-cognitive and non-conscious parts of a full consciousness system may help forge past earlier boundaries on the relation of consciousness and brain. 


\section{Lewis \& MacGregor}

Consciousness has long been recognized as being dependent on the well-being of the physical brain, and this is corroborated, for example, by its progressive degradation with brain loss in Alzheimer's disease. However, its association with particular structures (fixed or mobile as in a type of molecule or organelle) or functions or fields of the brain is problematical. Over the last half-century, it has been seen as particularly related to the neurobiology of the brain stem and arousal, the perihippocampal region and experiential memory, the cerebral cortex and thinking or higher sensory experience, the basal ganglia and cerebral cortex [22, 24]. Others have attributed consciousness to volume transmissions, extracellular space, or synaptic clefts [34, 35]. Recently, fMRI experimentation has suggested new ground in the possible particular relation of consciousness to metabolism or blood flow particularly as mediated by glutamate receptors on the astroglia cells, which exert local control on synapses in the dendrites of cortical pyramidal neurons $[25,36]$. It has been estimated that $80 \%$ of the brain's energy supply (in itself $\sim 20 \%$ of that of the entire body) is used by such receptors, particularly associated with NMDA synapses, which are thought to mediate plastic Hebbian learning. This indicates a major involvement with the patterned "inner constructions" [24, 35] of cortical neural networks. The striking idea of a primary association of consciousness with either metabolism or blood flow directly is consistent with the notable dependence of consciousness on brain oxygen and blood supply, and may help forge a higher perspective on the subject at hand. Metabolic and blood supply controls such as the glial glutamate receptors might qualify as indeterministic small number subsystems.

\subsection{Philosophical views of brain-consciousness relations}

Traditionally, philosophers have based their views regarding the nature of consciousness-brain relations on the contradiction between free will and the presumed strict determinism of physical science $[12,39]$. This has led to a split between deterministic monism and separative dualism. Deterministic monism sees free will as an illusion and consciousness as an ineffectual epiphenomenon. Separative dualism sees an obscure relationship between two distinct dimensions of reality, the physical and the experiential. Monists have historically tended to take the experiential as its cognitive and especially rational quality (mental and mind) and to dismiss its nonrational qualities as markedly less significant or subordinate. Dualists have tended to stress the experiential and humanist or religious values. This paper and other recent work strive to find non-traditional mechanisms to develop middle ground for consciousness-brain relations between these two poles, i.e., deterministic monism on the right, and separative dualism on the left. The discussion centers around indeterminism as a main non-traditional mechanism and the chaos model as its vehicle.

In the triumvirate model of consciousness, inner reflective space implies operational qualities of awareness itself and imagination, sensory experience includes rudimentary images and holistic perceptual integrations, free will includes the direction of attention and directed thinking, planning, and the initiation of actions. Any given 
instance of free will entail a holistic selective apprehension and intention, including an initiation of a selected action or a selection of a contingent guiding ground structure for actions. The brain's neurobiology can be seen to do all these operations within traditional physical science. It is only the conscious dimension itself and its compelling sense of free will that seem to be outside the pale.

\subsection{The indeterministic molecular chaos model}

The molecular chaos model in itself sees a macroscopic brain governed largely and holistically by a pseudo-deterministic physical law, except for the extensive and widespread elements of stochastic (indeterministic) small number elements throughout its neurophysiology and its neurobiological support systems. This model can be used to draw the picture of a brain conditioned by years of learning within its own changing universe of circumstances and stochastic personal trial-and-error learning to a highly unique set of stochastic inner structures which provide unique individuality of thinking, behaving, and responding in any given situation and which collectively appear to give all the observable external manifestations of free will.

For at least some monist theorists, such a model can be further taken as complete in itself, and to provide a completely self-contained materialistic view of brain function inclusive of what we perceive as free will. In this, consciousness is assumed to be associated with some as yet unidentified neural structures or features. A kind of "freedom" can be seen in this model in the stochastic range of state variable values obtaining at any given time in any given set of elements relevant to a particular situation, and its "free" selection to correspond to the set of states actually exhibited at this time. This kind of "freedom" of selection could operate at many levels throughout the brain in any given event. Overall behavior could be held in coherent bounds by embedding networks conditioned by much life experience. Small number elements could play a central part, and at some point, the overall selection would be expressed by a set of selected choices imposed in or by a number of such elements as a pattern corresponding to the effective sense of the selection.

It should be clearly recognized that this model, by attributing all of conscious selection and action to the natural integrative processes of the traditional physical brain, offers only a pseudo-free will, leaving our sense of true free will an illusion and consciousness an ineffectual epiphenomenon. If traditional physical brain operations do it all, then consciousness is ineffectual, an epiphenomenon which belies the existential sense of conscious free will. To go beyond this "deluded rider" syndrome, as we shall now label it, one needs to go past indeterminism in itself, as well as past traditional physical science to some additional non-traditional mechanism associated particularly to consciousness. Even a substantive identity of consciousness with traditional operative neural structures or features, which brings no additional (non-traditional) operative influences, leaves consciousness an epiphenomenon.

Nonetheless, this general chaos model, even with its deluded epiphenomenal consciousness is an appealing and plausible model with many adherents. A general form 
of it is introduced in the recent literature by Faure and Korn [13, 21]. This view is buttressed by clever experiments by Haggard and colleagues [28], which show that actions perceived to be initiated by the conscious mind can actually be initiated in the motor areas of the brain before the conscious mind perceives that it has made a decision. Such experiments may eventually give a Baconian/Lockean foundation to the question of free will. Some philosophers, especially Schopenhauer, emphasize the dominance of the will over the individual [45]. Many computational and other neuroscientists take similar monist views including the deluded rider implication [49]. The molecular chaos model is a new softened materially determinative but indeterministic monism, which can be seen as a moderate right view of consciousness-brain relations.

\subsection{Alternative modified monisms, localization and substantive identity}

The molecular chaos model may be seen as one of a class of mostly traditional rightish-middling views (depending on specifics in relating consciousness to selection and action), which see consciousness as somehow effecting selective choices in elements affecting networks for preparatory planning and direction, and initiation of expressive action, as integrated from the brain's neurophysiological flags and informational and other states [23]. As we have seen, any of these must invoke some further non-traditional mechanism beyond traditional physical science and indeterminism in itself to avoid the deluded rider syndrome.

The triumvirate model of unified consciousness (inner reflective space, sensory experience, and partially free will) suggests that as a consciousness system has multiple aspects. It is likely to have distributed association or localization across multiple physical structures or processes. Perhaps only some would be operationally associated with free will or indeterminism. The stochastic behavior of chaotic small number elements may help localize them and indeterminism in the brain.

Yet, the question of localization of consciousness depends ultimately on the larger question of whether and with what physical features or substance, if any, consciousness might be substantively identified or otherwise associated. Recent philosophy of mind has suggested alternative nonreductive materialist or nonreductive physicalist approaches to brain-mind relations, including concepts such as property dualism and double aspect theories $[5,6,9,10,39-42]$. One example is the idea that consciousness may be an emergent quasi-global quality that relates to some portion of brain physiology, the way temperature relates to the kinetics of a regional collection of molecules in a gas. Ultimately, any such hypothesis must in turn be able to answer the question, "if in this place and instance in the brain, why not elsewhere in or external to the brain?" This can help narrow the search for the neural foundations of consciousness by destroying the plausibility of most proposed identifications of consciousness to traditional physical structures or features, fixed or mobile, or, at best, 
pushing them to finer levels of identification. Others have drawn rather implausible conclusions of extraneural consciousness in robots and the like [24, 41].

\subsection{High level modulative energizing systems (global, extra-neural)}

What is needed to satisfy the conscious sense of free will is a substantive identity of consciousness with brain which includes a global sentience to the selection and formulative processes of the brain, the power to actively alter selected neurobiological states, and a self-referential sense of freedom of action. It seems plausible that all three of these might be satisfied by a global overall system, close to, but, outside of higher representational and semantic neural processing in themselves. This might be satisfied by either a diffusive neural system of networks or volume conductions. Alternative intriguing candidates for such global consciousness could involve the micro-local control of neural metabolism or blood supply by interactive neural/glial circuits, as suggested by neuroimaging techniques [25, 36]. Astrocytic networks could operate in continuous feedback association with appropriate neural and physiological signals throughout the brain to guide the direction of overall brain activity by selective distribution and redistribution of regional, local, and micro blood flow [24, 35]. Glutamate receptors might be a central ingredient. The grounding of consciousness with the fundamental energetic and life-supporting systems of the brain suggests a larger order of concerns and influences, offers the management of great complexity within an overall simplicity, has a credible existential feel and plausible operative relationship, localizes easier than full neurobiological complexity, and relates closely to the molding of the brain's inner constructions [24]. The system is governed by neural control systems, but also subject to additional biological physiology. Indeterministic small number processes could operate in its microphysiology.

Yet, if we see such a system as ultimately driven by traditional physics, then the role of its presumed consciousness is problematical, essentially unnecessary, still an epiphenomenon. Nonetheless, this view remains a plausible rightish materially-based possibility.

\subsection{Philosophical interlude}

Other theorists have argued that free will is too central a part of the fabric of human existence to be denied and that the idea of a deluded ineffectual epiphenomenal consciousness at the core of a robust vibrant species does not seem viable. Many, probably most, philosophers such as Bergson, have seen free will as a vital quality of human existence [4, 12]. Most non-western cultures have never doubted it [18, 46]. Existentially, the daily use of free conscious choice seems necessary for mere survival. One may regret, e.g., a passive giving over of one's active conscious self-direction to natural brain processes while trying to jay-walk through fast-moving traffic.

The deep structure of the consciousness-brain question is rooted in its dual aspects. The overwhelming quality of the brain for any human is the existential 
fact that one's conscious experiential existence appears its central operative core, and that one necessarily exerts partially free direction of internal and behavioral actions through it. One feels the validity of this in oneself, before and above all other things, except perhaps consciousness itself. Living and interacting with others, one naturally attributes and interrelates with an inferred kindred inner autonomous existential consciousness in others, and finds it very difficult to believe that the inner workings of humanity are not of this existential nature. The fact that consciousness is dependent on the physical well-being of the brain, and that certainly many and perhaps all of its operative properties have physical correlates in the brain, however, leads Baconian/Lockean natural science to try to understand consciousness completely within its general physical laws, as applied to the natural physical processes of the brain. This is a reductive approach which encounters difficulties regarding the correspondence of conscious free will with physical law, the nature of relational correspondence or the identity of consciousness with the physical structures or processes of the brain, the related question of localization of consciousness in the brain, and the comparative substantive natures of consciousness and physical matter. As individuals, philosophers, scientists, and lay thinkers each will have differing views on these matters. It is instructive to recall that reality may well require a larger rational understanding than our current scientific description of it, and perhaps even a larger scientific framework, if we are indeed to have a theory of everything.

\subsection{Emergent autonomous consciousness}

To avoid the deluded rider syndrome and explicitly represent free will in a brain governed by traditional physics, it is necessary to assume the operation of nontraditional mechanisms. There are two possibilities beyond but close to current scientific thinking. The first may be represented by the hypothesis of the emergence of a partially autonomous consciousness in close association with brain integration, as represented in the global modulative energizer outlined above. Such an emerging consciousness could have partial groundings in certain brain processes, yet attain unique autonomy with respect to known physical processes in selective interactive apprehension and activation of certain neural processes. This is a leftish middle ground which lends itself to the idea of the evolutionary emergence of consciousness within biological systems. It may or may not have a touch of dualism depending on its ultimate substantive nature, which is yet unknown.

\subsection{Energization and force in free will}

The possible non-traditional physical action of conscious free will to alter some neurobiological states (e.g., a selective choice within an indeterministic small number element) suggests that energization, i.e., the ability to exert a force, to bring about change, may possibly be the core of the consciousness-brain relationship. Natural forces in classical (macroscopic) physical science originate in and act on a specific 
quality of matter (which can be called a "charge") unique to that force (e.g., mass for gravitational force and electric charge for electromagnetic force). A force acting on an element in a triumvirate consciousness system (e.g., make a choice in or by an indeterministic small number element), might originate within the given element itself, within some region local or external to it, or within some kind of field distributed throughout the brain or the locale of the element, or all of these. A picture like this could be completed by the positing of a unique "charge" distributed through all or some subset of the members of the triumvirate. This charge could then define both the functional coherence and localization of the consciousness system and its effects. A picture like this could describe a consciousness system in terms of particles, charges, and forces underlying the spaces of conscious awareness and imagination, and its reflections, selections, initiations, passive images, and willful actions, and indeed, constitute an essential substantive identification of consciousness with the charge. Forces in the microscopic quantum mechanical physics of fundamental particles (which includes electrons and the like) also depend on the existence of charges, and occur as particle-carrier-charge complexes [15, 32].

\subsection{Quantum mechanical views}

Several authors have suggested roles for quantum mechanics in brain function [16, $24,30,37,48]$. An influence of quantum mechanical uncertainty would require the transmutation of that uncertainty to a microphysiological effect [24], either neural or supportive or both. Individual particles such as electrons or the like, perhaps housed in particular organelles or molecules, would be most likely. It is interesting that there may be considerable overlap between the indeterministic small number elements of the chaos model and these candidates for quantum mechanical influences. The strongest quantum mechanical hypothesis is that consciousness may correspond to a specific particle-force-charge complex. Such a model offers the possibility of a substantive identity of consciousness, which reduces the localization question to "if this occurs in human brain, where else might it occur?" This view is a unique central view positing substantive identity with explicit conscious and physical dimensions within the dualities of modern physics.

\subsection{Modified dualisms}

An alternative leftish-center idea is that consciousness is ultimately outside of physical science as it has been described so far, but capable of apprehending much of the brain's sensibilities and selectively altering its physiological states according to some specified physical action. Again, the chaotic small number elements could be involved. Such a view remains outside the Baconian/Lockian gates of natural science, unless natural science can be expanded to interrelate the experiential with the physical in a fundamental and verifiable way. In any case, such a view is close to the existential sense of any human. A more specific form of this idea has been 
suggested in the form that the "observer" of quantum mechanics can be seen as such a representation of consciousness and that the cite of selective action is in the quantum mechanical tunneling of electrons across synaptic clefts [48]. (The position of a single electron is a quantum mechanical state variable and this might also qualify as a small number element.)

\subsection{Summary of consciousness-brain models}

This section has defined six representative middling views between the classical polarity of deterministic monism and separative dualism: an indeterministic monism of the molecular chaos model, a high-level global extra-neural modulative energizing system, an emergent autonomous consciousness, a substantive quantum mechanical model, and two forms of physically-linked dualism.

The underlying obscurity in this array is the nature of the experiential dimension and its relation to the physical. Indeterminism in itself, as in the molecular chaos model, adds an apparent behavior quality which can be roughly interpreted as a pseudo-free will with a high degree of individuality and a mix of unpredictability within overall coherence similar to human behavior. It can be extended in the modulative energizer extension of the molecular chaos model to a further distancing from determinative neural processes and a different physiological grounding. Yet, any substantive identity of consciousness, which leaves all operative processes in traditional physical mechanisms, also leaves consciousness itself ineffectual, an epiphenomenon. Nonetheless, some will find this a believable representation of the fundamental nature of things and the human condition.

Others will feel that human free will requires a fuller expression than given by the indeterminism of the chaos model in itself, with its deluded epiphenomenal consciousness. The only two non-traditional properties utilized in the three remaining non-quantal, non-traditional consciousness-brain models are the abilities to sense some attributes of the brain integration, and to selectively alter some neurobiological states. The brain can do these things alone. There are only two reasons to want more. The first is the rejection of the deluded ineffectual epiphenomenal consciousness. This deluded rider violates the central existential sense of free will. It also seems to violate a basic principle of science, Ockham's razor, the principal of minimal sufficient hypothesis, which would seem to invert to "if consciousness is there it must do something beyond what the brain could do in its absence". The second is the recognition that "whatever causes consciousness in the brain must be unique to locations which might be reasonably thought to be also conscious" would seem to rule out a vast number of candidate substantive bases of consciousness.

The emergent autonomous consciousness model posits a minimal high-level agent of free selective choice in close interaction with the brain and perhaps brain-energy integration. The more exotic substantive quantum mechanical model posits a particular particle-carrier-charge complex for consciousness. It seems the most likely 
plausible direction available for this purpose is in the current physical theory. A larger leftish possibility is that natural science can be extended to include both consciousness and the physical within a larger rational fabric, closing in systematically on their relations and differences.

Dualist views more strongly separate the experiential and physical dimensions. Classical separative dualism sees consciousness as a separate substance distinct but parallel to matter, and associated particularly with an individual brain and able to apprehend much of its activity and influence it. The physically-linked dualisms narrow this by specifying explicit physical cites for the expressions of conscious free will.

All of the six views have some plausible grounding and appeal to one or another perspective of things. The underlying obscurity is the nature of the experiential dimension and its relation to the physical. The big breakthrough will be the unfolding of observational correspondences or perhaps substantive identity of consciousness with physical structures and processes of the brain. The stochastic behavior of small number elements throughout the brain predicted here may be a helpful clue in this direction. This problem area can be powerfully approached by neuroimaging techniques associated with penetrating subassembly modeling both in humans [36], and with microelectrodes added, in animals.

In the last analysis, any particular assumed association between consciousness and neurobiology reduces to an apparent correspondence. At this stage of our collective understanding, we have at best only speculative representations on both sides of a textural-modal divide standing in presumed association with each other. In this paper, we have assumed that the physical correlates of consciousness suggested throughout the work would influence whatever biological systems in which they occur. It is plausible that different levels of systemic biological, perhaps physiological, organization themselves play the decisive role in the identification of consciousness in conjunction with, or even rather than, the explicitly physical bases we have emphasized here. Only further investigation can clarify this. Certainly systemic biological organization of the brain is central to the subject. All thoughts about the foundations of consciousness must ultimately be related to these. Moreover, it is very difficult to pass mechanistically from the bottom up from physics through biology, let alone on to consciousness. Biology itself is much less systematic than physics. Gilbert Chauvet has recently provided a mathematical formalism to undertake the first step in such a process $[7,8]$. Theoretical and integrative neuroscience and related philosophy of mind can help inform the general unfolding of this large difficult, rich, multifaceted and vitally important area of brain research. We hope that this paper will encourage more active participation by neuroscientists on all these topics.

It is significant that the indeterminism of small number particle systems, the chaos model, and the several "middle ground views" discussed here, both undermine the widely-held assumption of brain determinism [20] and further weaken and restrict the popular computational metaphor of brain function [43] which has 
previously been discredited as biologically overreductive, implausible, and overreaching $[24,34,41]$.

\subsection{Indeterminism revisited}

This paper has presented grounds for stating that macroscopic behavior in the brain and in the physical universe as a whole is certainly observationally indeterministic, as manifested particularly within small number elements, and may be both observationally and intrinsically indeterministic. This small number indeterminism has been shown to contribute significantly to neural activity and to several plausible views of consciousness-brain relations.

It is notable that both small number situations and the other central forms of indeterminism in physics, quantum mechanics, point to overlapping sets of candidate brain elements for indeterminism. The degree of this overlap needs to be further explored. Since the natural domain of quantum mechanical effects $[15,32]$ is smaller in scale, it is possible that the small number elements magnify and transmute quantum variability to effective neurobiological levels. Since the overall Schrödinger wave function for a given system progresses deterministically between periods of observation, future work could use it for a simple model system to corroborate the growth of uncertainty for the smaller ends of this work's macroscopic estimations, and to inform this possibility that macroscopic indeterminism may be partially initiated by intrinsic quantum uncertainty.

It is interesting that the mix of indeterminism within an overall loose general directive determinism, suggested by the chaos model of the brain, is similar to that prescribed for the universe by quantum mechanics. Since the Schrödinger probability functions for the state variables of quantum particles evolve deterministically even though their exact values can never be simultaneously fixed, the likelihood of future events could be predicted by a sufficiently vast intelligence, even though no occurrences can be exactly predicted. (This is controversial. Some think that the total deterministic evolution of wave forms is lost by the absorption of some particles in black holes.)

Just as the supposition of macroscopic determinism is an article of faith, so is the supposition of intrinsic macroscopic indeterminism, even in the certainly observationally indeterministic small number elements. Perhaps underlying any observational indeterminism in macroscopic systems, the traditional underlying determinism does apply and would be knowable to the classical "sufficiently vast intelligence", even if we humans may never be able to measure or prove it. Nonethe-

less, the practical observational stochastic nature of small number systems seems a certain and central quality of brain function.

It is not clear that free will need to be dependent on indeterminism. This section has argued that indeterminism is not sufficient for a consciously-driven free will, but requires an additional non-traditional mechanism. Yet, in these latter cases, indeterminism may not be necessary. A non-traditional willful action or selection 
might be effective in a deterministic system, though likely less so. Mechanistically, it is interesting that, while quantum particles are never completely defined but smeared over ranges of values of their state variables at all times, macroscopic particles of even indeterministic small number elements are always at some particular state point, whether we can ever know or predict it or not.

Nonetheless, the considerations of this section clearly support the idea that indeterminism is a highly useful quality in models relating to free will and in brain operations generally. Small number circumstances seem to apply to vast numbers of instances involving receptor molecules, synaptic clefts, and other microphysiological cites where single particles are closely linked to neural or neural supportive operations.

\section{Acknowledgments}

The two-body gedanken model presented in this paper was inspired by a problem assigned to students in an upper-division quantum-mechanics course taken by one of us many years ago. The problem involved invoked the uncertainty principle to estimate the maximum number of times a ping-pong ball, dropped as precisely as possible, could be made to bounce from the sharpened tip of a pencil that was fixed in space.

We thank World Scientific Publishing Co. for permission to draw from Chaps. 1 and 6 of On the Contexts of Things Human — an Integrative view of Consciousness, Brain, and Freedom of will, 2006 by MacGregor RJ. See also website http://www.worldscibooks.com/lifesci/6073.html.

\section{References}

[1] Baylor DA, Lamb TD, Yau KW, Response of retinal rods to single photons, J PhysiolLondon 288:613-634, 1979.

[2] Barlow HB, Levick WR, Three factors limiting the reliable detection of light by retinal ganglion cells of the cat, $J$ Physiol-London 200:1-24, 1969.

[3] Barlow HB, Levick WR, Yoon M, Responses to single quanta of light in retinal ganglion cells of the cat, Vision Res Suppl 3:87-101, 1971.

[4] Bergson H, Time and Free Will, Pogson F, (Tr.) (ed.), Macmillan, New York, 1919.

[5] Chalmers DJ, The Conscious Mind: In Search of a Fundamental Theory, Oxford University Press, New York, 1996.

[6] Chalmers DJ (ed.), Philosophy of Mind: Classical and Contemporary Readings, Oxford University Press, Oxford, 2002.

[7] Chauvet GA, Theoretical Systems in Biology: Hierarchical and Functional Integration, Pergamon Press, Oxford, 1996.

[8] Chauvet GA, On the mathematical integration of the nervous tissue based on the s-propagator formalism: I. Theory, J Integr Neurosci 1:31-68, 2002.

[9] Churchland PS, Neurophilosophy, MIT Press, Cambridge, MA, 1986. 
[10] Churchland PS, Brain-Wise: Studies in Neurophilosophy, MIT Press, Cambridge, MA, 2002.

[11] Cohen IB, Whitman A, Isaac Newton: The Principia, University of California Press, Berkeley, 1999.

[12] Edwards P (ed.), The Encyclopedia of Philosophy, Macmillan Pub Co, NY, 1967.

[13] Faure P, Korn H, Is there chaos in the brain? I. Concepts of non-linear dynamics and methods of investigation, C R Biol 324:773-793, 2001.

[14] Green DM, Swets JA, Signal Detection Theory and Psychophysics, Wiley, New York, 1966.

[15] Greene B, The Elegant Universe, W. W. Norton, New York, 1999.

[16] Hameroff SR, Quantum coherence in microtubules: A neural basis for emergent consciousness? J Consc Studies 1:98-118, 1994.

[17] Hecht S, Shaer S, Pirenne MH, Energy quanta and vision, J Opt Soc Am 38:196-208, 1942.

[18] Higgins KM, World Philosophy, The Teaching Company, Chantilly, VA, 2001.

[19] Jacobson TA, Perentani R, An echo of black holes, Sci Am 293:69-75, 2005.

[20] Koch C, Hepp K, Quantum mechanics in the brain, Nature 440:611-612, 2006.

[21] Korn H, Faure P, Is there chaos in the brain? II. Experimental evidence and related models, C R Biol 326:787-840, 2003.

[22] MacGregor RJ, Theoretical Mechanics of Biological Neural Networks, Academic Press, Boston, 1993.

[23] MacGregor RJ, A functional theory of consciousness and its relations in brain, J Integr Neurosci 3:23-29, 2004.

[24] MacGregor RJ, On the Contexts of Things Human - An Integrative View of Consciousness, Brain, and Freedom of Will, World Scientific, Singapore, 2006.

[25] Magistretti PJ, Pellerin L, Cellular mechanisms of brain energy metabolism and their relevance to functional brain imaging, Philos T Roy Soc B 354:1155-1163, 1999.

[26] Maldacena J, The illusion of gravity, Sci Am 293:57-63, 2005.

[27] Minor AV, Kaisling KE, Cell responses to single pheromone molecules may reflect the activation kinetics of olfactory receptor molecules, J Comp Physiol A 189:221-230, 2003.

[28] Obhi SS, Haggard P, Free will and free won't, Am Sci 92:358-365, 2004.

[29] Peirce CS, Collected Papers of Charles Sanders Peirce, Cambridge, Harvard Univ Press, $1867 / 1960$.

[30] Penrose R, Shadows of the Mind: A Search for the Missing Science of Consciousness, Oxford Univ Press, 1994.

[31] Platt JR, Strong inference, Science 146:347-353, 1964.

[32] Pollack S, Particle Physics for Non-Physicists, The Teaching Co, Chantilly, VA, 2003.

[33] Popper KR, Conjectures and Refutations: The Growth of Scientific Knowledge, Routledge \& K Paul, London, 1963.

[34] Poznanski RR, Introduction to Integrative Neuroscience, in Poznanski RR (ed.), Biophysical Neural Networks: Foundations of Integrative Neuroscience, Mary Ann Liebert, New York, 2001.

[35] Poznanski RR, Towards an integrative theory of cognition, J Integr Neurosci 1:145-156, 2002. 
June 3, $2006 \quad$ 13:7 WSPC/179-JIN 00111

On Indeterminism, Chaos, and Small Number Particle Systems in the Brain 247

[36] Poznanski RR, Riera J, fMRI Models of dendritic and astrocytic networks, J Integr Neurosci 5, 2006.

[37] Ramon-Moliner E, The Conscious States of Matter, Vantage Press, New York, 1994.

[38] Schneider D, 100 years of pheromone research, Naturwissenschaften 79:241-250, 1992.

[39] Searle JR, The Rediscovery of the Mind, MIT Press, Cambridge, MA, 1992.

[40] Searle JR, The Mystery of Consciousness, I 85 II, New York Review of Books, 1995.

[41] Searle JR, Consciousness and the Philosophers, New York Review of Books, 1997.

[42] Searle JR, Consciousness, Ann Rev Neurosci 2:557-578, 2000.

[43] Sejnowski TJ, Koch C, Churchland PS, Computational neuroscience, Science 241:1299-1306, 1988.

[44] Shawhan PS, Gravitational waves and the effort to detect them, Am Sci 92:350-357, 2004.

[45] Shopenhauer W, The World as Will and Representation, Payne EFJ (Tr.) (ed.), 2 vols., New York, 1818, 1844.

[46] Solomon RC, Higgins KM, A Short History of Philosophy, Oxford University Press, New York, 1996.

[47] Sommerfeld A, Thermodynamics and Statistical Mechanics, Academic Press, New York, 1956.

[48] Walker EH, The Physics of Consciousness, Perseus Press, NY, 2000.

[49] Wegner MD, The Illusion of Conscious Will, MIT Press, Cambridge, MA, 2003. 
June 3, $2006 \quad$ 13:7 WSPC/179-JIN 00111 